\title{
Escalado temporal en agregación de partículas coloidales recubiertas con BSA
}

\author{
A. SCHMITT ${ }^{1}$, M. FERNÁNDEZ-BARBERO ${ }^{2}$, A. CABRERIZO-VÍlCHEZ ${ }^{1}$ y R. HIDALGO-ÁLVAREZ ${ }^{1}$ \\ ${ }^{1}$ Departamento de Física Aplicada, Facultad de Ciencias, \\ Universidad de Granada, Campus de Fuentenueva, E-18071 Granada, España. \\ ${ }^{2}$ Departamento de Física Aplicada, Universidad de Almería, Cañada de San Urbano s/n, E-04120 Almería, España.
}

\begin{abstract}
Este trabajo presenta un estudio experimental de la agregación de partículas de poliestireno cuyas propiedades superficiales se alteraron mediante la adsorción de distintas cantidades de Albúmina del Suero Bovino (BSA). La técnica de detección individualizada de la intensidad de la luz dispersada por una partícula (Single Cluster Light Scattering, SCLS) se empleó para el seguimiento de los procesos de agregación inducidos a alta concentración de electrolito. Empleando la teoría de escalado dinámico, se desarrolla un método para determinar el coeficiente de homogeneidad $\lambda$ a partir de la evolución temporal de la distribución de tamaños y del tamaño medio de los agregados. Se observa que, bajo las condiciones experimentales de este trabajo, el parámetro $\lambda$ es aproximadamente cero y prácticamente independiente del grado de recubrimiento de las partículas. Esto significa que las muestras están agregando en el régimen de agregación limitada por difusión. Únicamente para partículas completamente recubiertas se observa un ligero incremento en el valor de $\lambda$ que indica que el proceso de agregación se está alejando del régimen de agregación limitada por difusión.
\end{abstract}

Palabras clave: agregación coloidal, escalado dinámico, detección individualizada de partículas, adsorción de proteínas.

\section{Dynamic Scaling in Aggregation of BSA Covered Colloidal Particles}

In this work, an experimental study on aggregation processes of surface modified polystyrene particles is present. The particle surface characteristics were altered by adsorbing different amounts of Bovine Serum Albumin (BSA). Single Cluster Light Scattering (SCLS) was employed to monitor aggregation processes induced at high salt concentration. Based on dynamic scaling properties, a method is developed which allows the homogeneity exponent $\lambda$ to be obtained directly from the time evolution of the cluster size distribution and the average mean cluster size. Under the given experimental conditions, the homogeneity exponent $\lambda$ was observed to be approximately zero and practically independent of the degree of surface coverage of the particles. This means that the samples aggregate in the diffusion limited aggregation regime. Only for the completely covered particles, a small increase for $\lambda$ was observed which indicates that the corresponding aggregation process is drifting away from pure diffusion limited aggregation.

Keywords: colloidal aggregation, dynamic scaling, single cluster light scattering, protein adsorption.

\section{INTRODUCCIÓN}

La agregación coloidal se puede considerar como un modelo ideal para el estudio de procesos de crecimiento y formación de estructuras. En estos procesos, subunidades de menor tamaño se acercan debido al movimiento difusivo para luego unirse y formar agregados de tamaño mayor.

La velocidad de agregación depende no solo de la probabilidad de colisión entre dos partículas sino también de la probabilidad para la formación de una unión estable entre ellos. El régimen de agregación controlada por difusión (DLCA) se alcanza cuando todas las colisiones entre dos agregados conducen a la formación de un enlace. Una muestra coloidal agrega en el régimen de agregación controlada por reacción (RLCA) cuando la probabilidad de unión es mucho menor que la unidad. Existe una zona de transición entre los regímenes de DLCA y RLCA que no está bien delimitada.

Generalmente, la probabilidad de unión entre dos agregados depende de las características superficiales de los mis- mos. En suspensiones acuosas, las partículas presentan grupos químicos en su superficie cuya carga depende del $\mathrm{pH}$ de la fase acuosa. Normalmente, las fuerzas electrostáticas contrarrestan las fuerzas atractivas de tipo London-van der Waals y las partículas se mantienen en suspensión. La agregación comienza si la carga eléctrica se anula o se apantalla cambiando el $\mathrm{pH}$ o añadiendo electrolitos a la fase acuosa, respectivamente.

Otra posibilidad de modificar las características superficiales de las partículas consiste en la adsorción de macromoléculas. En función del grado de recubrimiento y de las propiedades electroquímicas de la fase acuosa puede observarse una amplia gama de fenómenos como la agregación limitada por reacción (RLCA), la agregación por puenteo y la estabilización estérica $(1,2)$.

En este trabajo, se estudia la influencia de las propiedades superficiales en la agregación coloidal mediante la técnica de detección individualizada de la intensidad de la luz dispersa- 
da por una partícula (Single Cluster Light Scattering, SCLS). Esta técnica permite el seguimiento de la distribución de tamaños y del tamaño medio de los agregados $\left\langle\mathrm{n}_{\mathrm{n}}\right\rangle$. A partir de estos datos, se obtienen las funciones de escalado $s(t)$ y $\Phi(\mathrm{x})$ mediante un procedimiento de ajuste.

\section{TEORÍA}

En sistemas diluidos, los fenómenos de agregación se pueden esquematizar de la manera siguiente (3):

$$
(\mathrm{i}-\text { mero })+(\mathrm{j}-\text { mero }) \underset{\mathrm{k}_{\mathrm{ij}}}{\longrightarrow}(\mathrm{i}+\mathrm{j})-\text { mero }
$$

En este esquema, la expresión "i-mero" representa un agregado formado por un número i de subunidades idénticas. La constante, $\mathrm{k}_{\mathrm{ij}}=\mathrm{k}_{\mathrm{ji}} \geq 0$, cuantifica la velocidad de reacción entre los i-meros y j-meros. El conjunto de constantes $\mathrm{k}_{\mathrm{ij}}$ se denomina kernel de reacción y contiene toda la información física sobre el proceso de agregación.

La ecuación de Smoluchowski (4):

$$
\frac{d}{d t} c_{n}=\frac{1}{2} \sum_{i+j=n} k_{i j} c_{i} c_{j}-c_{n} \sum_{i=1}^{\infty} k_{n i} c_{i}
$$

describe la evolución temporal de la distribución de tamaños, $\mathrm{c}_{\mathrm{n}}(\mathrm{t})$, en términos del kernel de reacción $\mathrm{k}_{\mathrm{ij}}$. Esta ecuación considera únicamente colisiones binarias que pueden aumentar o disminuir la concentración $\mathrm{c}_{\mathrm{n}}$ de un agregado de tamaño n. El primer término del miembro derecho corresponde a la formación de un n-mero a partir de un agregado de tamaño i y otro de tamaño (n-i). El segundo término del miembro derecho representa la pérdida de n-meros cuando estos chocan con otros agregados para formar agregados de mayor tamaño.

A pesar de su forma sencilla, no existe una solución analítica global de la ecuación de Smoluchowski. Únicamente en algunos casos especiales, como el kernel constante $\left(\mathrm{k}_{\mathrm{ij}}=\mathrm{k}_{11}=\right.$ cte.), el kernel suma $\left(\mathrm{k}_{\mathrm{ij}}=\mathrm{k}_{11}(\mathrm{i}+\mathrm{j}) / 2\right)$ y el kernel producto $\left(\mathrm{k}_{\mathrm{ij}}=\mathrm{k}_{11} \mathrm{ij}\right)$ entre otros, se conoce la solución analítica explícitamente. Para el kernel constante y condiciones iniciales monoméricas $\left(c_{n}(t=0)\right.$ $=\mathrm{c}_{0} \delta_{1 \mathrm{n}}$ ), la distribución de tamaños viene dada por:

$$
c_{n}=c_{0} \frac{\left(\frac{c_{0} k_{11}}{2} t\right)^{n-1}}{\left(1+\frac{c_{0} k_{11}}{2} t\right)^{n+1}}
$$

donde $\mathrm{c}_{0}$ es la concentración inicial de partículas monoméricas. $\delta_{x y}$ es el símbolo de Kronecker.

Van Dongen y Ernst introdujeron un esquema para clasificar kernels homogéneos en términos de dos parámetros, $\lambda$ y $\mu$, según (5)

$$
\begin{array}{lc}
\mathrm{k}_{(\mathrm{aii})(\mathrm{aj})} \approx \mathrm{a}^{\lambda} \mathrm{k}_{\mathrm{ij}} & \lambda \leq 2 \\
\mathrm{k}_{\mathrm{i}<<\mathrm{j}} \approx \mathrm{k}_{0} \mathrm{i}^{\mu} \mathrm{j}^{(\lambda-\mu)} & \lambda-\mu \leq 1
\end{array}
$$

donde la constante a es un número positivo grande $\mathrm{y}_{0}$ un factor de escala.

Para kernels que no dan lugar a la formación de una fase gel $(\lambda \leq 1)$, la distribución de tamaños $c_{n}(t)$ se pude expresar en términos de dos funciones $\mathrm{s}(\mathrm{t})$ y $\Phi(\mathrm{x})$ según $(3,5,6)$

$$
\mathrm{c}_{\mathrm{n}}(\mathrm{t}) \sim \mathrm{s}^{-2} \Phi(\mathrm{n} / \mathrm{s})
$$

La función $\mathrm{s}(\mathrm{t})$ está relacionada con el tamaño medio de los agregados. La función $\Phi(\mathrm{x})$ puede interpretarse como una distribución de escalado del tamaño. $\Phi(\mathrm{x})$ es independiente del tiempo y, por lo tanto, caracteriza un proceso de agregación dado. Ha de destacarse que la relación [5] es estrictamente válida sólo para agregados grandes y tiempos largos. No obstante, en muchos sistemas se encontró que las distribuciones de tamaño alcanzan el límite de escalado dinámico, representado por la ecuación [5], también para agregados pequeños y a tiempos relativamente cortos $(3,7)$.

Para kernels homogéneos, la solución analítica para la función $\mathrm{s}(\mathrm{t})$ viene dada por [8]

$$
\mathrm{s}(\mathrm{t})=\left\{\begin{array}{cc}
{\left[\mathrm{C}_{1}+(1-\lambda) \mathrm{C}_{2} \mathrm{t}\right]^{1 / 1-\lambda}} & \lambda<1 \\
\mathrm{C}_{3} \exp \left(\mathrm{C}_{2} \mathrm{t}\right) & \lambda=1
\end{array}\right.
$$

donde $\mathrm{C}_{1}, \mathrm{C}_{2}$ y $\mathrm{C}_{3}$ son constantes que dependen de las condiciones iniciales. Obsérvese que a tiempos largos, la función $\mathrm{s}(\mathrm{t})$ crece como una potencia del tiempo para kernels con $\lambda<1$,

$$
S \sim t^{1 / 1-\lambda}
$$

De la distribución de escalado del tamaño, $\Phi(\mathrm{x})$, se conoce únicamente el comportamiento límite para argumentos grbindes y pequeños (8)

$$
\begin{aligned}
& \Phi(\mathrm{x}>>1) \sim \mathrm{x}^{-\lambda} \exp \left(-C_{4} \mathrm{x}\right) \quad 0 \leq(\lambda-\mu)<1 \\
& \Phi(\mathrm{x} \ll 1) \sim\left\{\begin{array}{lcc}
\mathrm{x}^{-\tau} \exp \left(-\mathrm{C}_{5} \mathrm{x}^{-|\mu|}+\ldots\right) & \tau \text { depende del kernel } & \mu<0 \\
\mathrm{x}^{-\tau} & \tau<(\lambda+1) & \mu=0 \\
\mathrm{x}^{-\tau} & \tau=\lambda+1 & \mu>0
\end{array}\right.
\end{aligned}
$$

En estas ecuaciones, $\mathrm{C}_{4}$ y $\mathrm{C}_{5}$ son constantes. $\tau$ es un exponente desconocido cuyo valor depende de la forma concreta del kernel.

En algunos casos especiales, se conoce la relación exacta entre la función $\mathrm{s}(\mathrm{t}) \mathrm{y}$ el tamaño medio $<\mathrm{n}_{\mathrm{n}}>$ de los agregados (8).

$$
\mathrm{s}(\mathrm{t}) \sim\left\{\begin{array}{lc}
\left\langle\mathrm{n}_{\mathrm{n}}\right\rangle & (\tau<1) \quad \text { ó } \quad(\mu<0) \\
\left\langle\mathrm{n}_{\mathrm{n}}\right\rangle^{1 /(2-\tau)} & 1<\tau<2
\end{array}\right.
$$

Para procesos de agregación controlados por difusión la función $\mathrm{s}(\mathrm{t})$ es directamente proporcional al tamaño medio $<\mathrm{n}_{\mathrm{n}}>$. Para otros procesos de agregación, no se conoce a priori la relación entre $\mathrm{s}(\mathrm{t}) \mathrm{y}<\mathrm{n}_{\mathrm{n}}>$.

No obstante, la ecuación [10] sugiere que la función $\mathrm{s}(\mathrm{t})$ viene generalmente dada como potencia del tamaño medio $<\mathrm{n}_{\mathrm{n}}>$, es decir como

$$
\mathrm{s}(\mathrm{t}) \sim\left\langle\mathrm{n}_{\mathrm{n}}\right\rangle^{\alpha}
$$

donde $\alpha \geq 1$. El exponente $\alpha$ se puede determinar experimentalmente dado que la distribución de tamaños $c_{n}(t)$ alcanza su 
límite de escalado dinámico únicamente con la función $\mathrm{s}(\mathrm{t})$ correctamente elegida (9). Para ello, se calcula la distribución de escalado, $\Phi(\mathrm{x})$, según la ecuación (5) para distintos valores de $\alpha$. Únicamente con el valor correcto de $\alpha$, la distribución de tamaños colapsa en una sola curva maestra y define la función $\Phi(\mathrm{x})$.

Una vez conocido el valor del exponente $\alpha$, se puede proceder al calculo del parámetro de homogeneidad $\alpha$. Combinando la ecuación [7] con la ecuación [11], se obtiene

$$
\left\langle n_{n}\right\rangle \sim \mathrm{t}^{1 / \alpha(1-\lambda)} \sim \mathrm{t}^{\mathrm{w}}
$$

de donde se obtiene el exponente $\lambda$

$$
\lambda=1-\frac{1}{\alpha w}
$$

\section{PROCEDIMIENTO EXPERIMENTAL}

En este trabajo, se empleó como sistema coloidal modelo la muestra AS8 formada por una suspensión acuosa de partículas esféricas y monodispersas de poliestireno. El diámetro de las partículas fue de $(580 \pm 27) \mathrm{nm}$ con un índice de polidispersidad de 1.005. La carga superficial de las partículas, proveniente de grupos sulfato, fue de $(-2.4 \pm 0.1) \mu \mathrm{C} / \mathrm{cm}^{2}$. Estas características se encuentran resumidas en la tabla I.

Las propiedades superficiales de las partículas AS8 se modificaron mediante la adsorción de distintas cantidades de la Albúmina del Suero Bovino (BSA). Añadiendo una cantidad dada de BSA a una muestra estable de AS8, se prepararon partículas con distintos grados de recubrimiento con esta macromolécula.

La temperatura y la concentración iónica de la fase acuosa durante los experimentos de agregación fueron de $(21 \pm 1)^{\circ} \mathrm{C}$ y $1.0 \mathrm{M}$ de $\mathrm{KCl}$, respectivamente. La concentración inicial de partículas fue de $1.0 \times 10^{8} \mathrm{~cm}^{-3}$. El pH de la fase acuosa se fijo en 5.0 mediante un tampón de acetato.

Para el seguimiento de los distintos proceso de agregación se empleó un dispositivo óptico de detección individualizada de partículas (SCLS, Single Cluster Light Scattering). Está técnica se basa en la medición de la intensidad de la luz dispersada por una partícula a bajo ángulo que, por lo tanto, permite contar y clasificar las partículas según su tamaño. El dispositivo experimental registra la distribución de tamaños hasta heptámeros y el número total de partículas como función del tiempo. Para más detalles véase $(7,10,11)$.

\section{RESULTADOS EXPERIMENTALES}

En este trabajo, se estudiaron los procesos de agregación para la muestra AS8 con un $0 \%, 25 \%, 50 \%, 75 \%$ y $100 \%$ de la superficie de las partículas recubiertas con BSA. La agregación se indujo mezclando volúmenes iguales de muestra y electrolito al doble de la concentración final deseada. Las medidas de la distribución de tamaño de los agregados y del número total de agregados se realizaron mediante SCLS. El tamaño medio $\left\langle\mathrm{n}_{\mathrm{n}}\right\rangle$ de los agregados se calculó como cociente entre el número inicial de partículas monoméricas y el número total de agregados.

En primer lugar se determinaron las constantes de velocidad de agregación para los cinco recubrimientos estudiados.
TABla I: CARACTERÍSTICAS DE LAS PARTíCULAS DE POLIESTIRENO.

\begin{tabular}{|c|c|c|c|c|}
\hline forma & $\begin{array}{c}\text { diámetro } \\
(\mathrm{nm})\end{array}$ & $\begin{array}{c}\text { índice } \\
\text { polidispersidad } \\
\text { (i.d.p.) }\end{array}$ & $\begin{array}{c}\text { grupos } \\
\text { superficiales }\end{array}$ & $\begin{array}{c}\text { densidad de } \\
\text { carga superficial } \\
\left(\mu \mathrm{C} / \mathrm{cm}^{2}\right)\end{array}$ \\
\hline esférica & $580 \pm 27$ & 1.005 & sulfato & $-2.4 \pm 0.1$ \\
\hline
\end{tabular}

TABLA II: CONSTANTE DE VELOCIDAD K11 Y EXPONENTES CARACTERÍSTICOS DEL ESCALADO DINÁMICO COMO FUNCIÓN DEL RECUBRIMIENTO.

\begin{tabular}{|c|c|c|c|c|c|}
\hline recubrimiento (\%) & $\mathbf{0}$ & $\mathbf{2 5}$ & $\mathbf{5 0}$ & $\mathbf{7 5}$ & $\mathbf{1 0 0}$ \\
\hline $\mathrm{k}_{11}\left(10^{-12} \mathrm{~cm}^{3} / \mathrm{s}\right)$ & $7.3 \pm 1.8$ & $6.2 \pm 1.5$ & $5.8 \pm 1.4$ & $3.6 \pm 0.9$ & $1.6 \pm 0.4$ \\
\hline $\mathrm{w}$ & $1.1 \pm 0.1$ & $0.9 \pm 0.1$ & $0.8 \pm 0.1$ & $0.7 \pm 0.1$ & $0.55 \pm 0.05$ \\
\hline$\alpha$ & $1.0 \pm 0.1$ & $1.2 \pm 0.1$ & $1.3 \pm 0.1$ & $1.5 \pm 0.2$ & $2.3 \pm 0.2$ \\
\hline$\lambda$ & $0.09 \pm 0.18$ & $0.07 \pm 0.19$ & $0.04 \pm 0.19$ & $0.05 \pm 0.19$ & $0.21 \pm 0.16$ \\
\hline
\end{tabular}

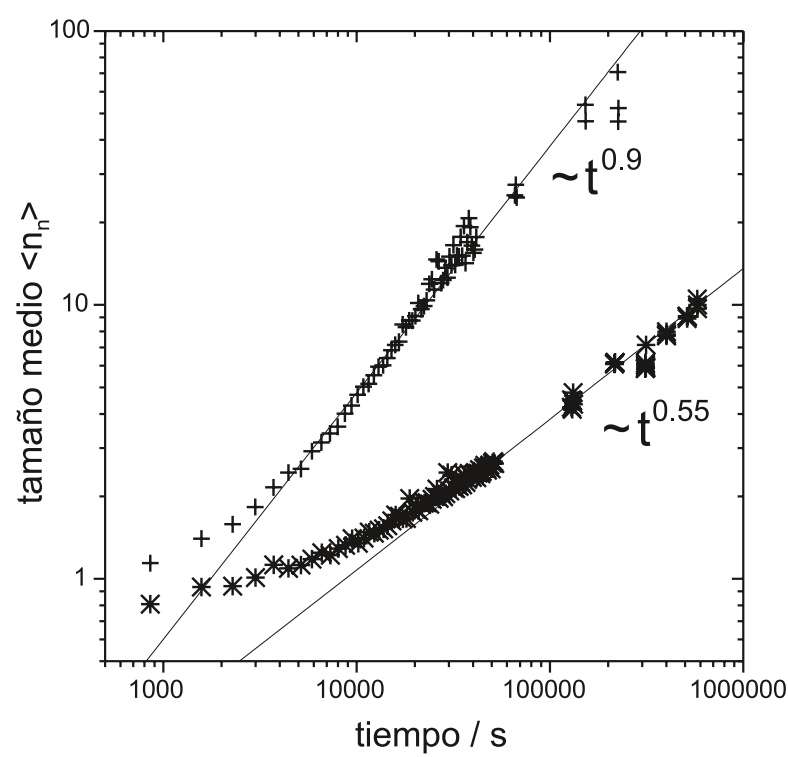

Tamaño medio en numero, $<\mathrm{n}_{\mathrm{n}}>$, como función del tiempo en escala logarítmica. Los símbolos $(+)$ y $\left(^{*}\right)$ corresponden a los grados de recubrimiento de $25 \%$ y $100 \%$, respectivamente. Las líneas continuas representan el comportamiento límite $\left\langle\mathrm{n}_{\mathrm{n}}>\sim \mathrm{t}^{\mathrm{w}}\right.$ para tiempos largos.

Para ello, se representó la inversa de la raíz cuadrada de la concentración de monómeros como función del tiempo. Para este tipo de representación, se espera un comportamiento lineal si las muestras agregan según el modelo del kernel constante (ver ecuación [3]). En todos los casos, se observó una dependencia lineal por lo menos durante los primeros instantes de agregación. A partir de las pendientes de las rectas ajustadas se calculó la constante de velocidad $\mathrm{k}_{11}$.

La tabla II recoge los resultados obtenidos para todos los grados de recubrimiento estudiados. Se observa que la velocidad decrece conforme aumenta el grado de recubrimiento. Bajo las condiciones experimentales descritas anteriormente, se espera que las partículas sin proteína adherida en su superficie, agreguen en el régimen de agregación limitada por difusión. El valor experimental de la constante de velocidad, $\mathrm{k}_{11}$, obtenido para este caso es de aproximadamente $7 \times 10^{-12} \mathrm{~cm}^{3} / \mathrm{s}$. Este valor concuerda perfectamente con el valor medio de $(6 \pm 3) \times 10^{-12} \mathrm{~cm}^{3} / \mathrm{s}$ calculado por Sonntag y Strenge para muestras que agregan bajo condiciones de DLCA (12). 
Para las partículas con un grado de recubrimiento bajo e intermedio, se espera una aportación del mecanismo del puenteo al proceso de agregación. Las constantes experimentales de velocidad observadas en este caso no difieren significativamente de las constantes observadas para las partículas no recubiertas. Esto demuestra que también en este caso, la agregación está limitada únicamente por la difusión de los agregados y todas las colisiones entre dos agregados en configuración de puenteo deben conducir a la formación de un enlace estable.

El descenso drástico en la constante de velocidad para recubrimientos por encima del $50 \%$ se puede explicar en términos de efectos estéricos que impiden que dos partículas totalmente recubiertas con proteínas formen una unión estable entre sí. En este caso, la agregación se aleja del régimen de agregación limitada por difusión.

La segunda parte de este trabajo consiste en la obtención del parámetro de homogeneidad $\lambda$ que caracteriza los regímenes de agregación de una forma más directa. Para ello se representó el tamaño medio de los agregados como función del tiempo en escala logarítmica. La figura 1 muestra esta representación para los grados de recubrimiento de $25 \%$ y $100 \%$. Se observa claramente, que el tamaño medio $<\mathrm{n}_{\mathrm{n}}>$ alcanza un comportamiento límite en forma de ley de potencia en ambos casos. Para los demás grados de recubrimiento, se obtuvo un comportamiento similar. La tabla 2 recoge los exponentes $\mathrm{w}$ ajustados según la ecuación [12]. Los valores obtenidos decrecen desde la unidad hasta aproximadamente un medio conforme aumenta el grado de recubrimiento de los agregados.

Como siguiente paso para la obtención del parámetro de homogeneidad $\lambda$, se calculó la distribución de escalado $\Phi(\mathrm{x})$ a partir de la distribución de tamaños $c_{n}(t)$ y el tamaño medio $<\mathrm{n}_{\mathrm{n}}>(\mathrm{t})$ mediante las ecuaciones [5] y [11]. El exponente $\alpha$ se empleó como parámetro de ajuste y se variaba hasta que todas las curvas de la distribución definían una única curva maestra. La tabla II recoge los resultados obtenidos para el exponente $\alpha$. Se puede observar que $\alpha$ aumenta gradualmente con el grado de recubrimiento. Para las partículas no recubiertas, se obtiene el valor esperado de $\alpha=(1.0 \pm 0.1)$ mientras que en el caso de las partículas totalmente recubiertas, el valor obtenido es de $(2.3 \pm 0.2)$. Broide y cols. observan $\left.\mathrm{s}(\mathrm{t}) \sim<\mathrm{n}_{\mathrm{n}}\right\rangle$, es decir $\alpha=1$, en el caso de DLCA y $\left.\mathrm{s}(\mathrm{t}) \sim<\mathrm{n}_{\mathrm{n}}\right\rangle^{2}$, es decir $\alpha=$ 2, en el caso de la agregación lenta [8]. Esta observación parece indicar que se está produciendo una transición de agregación limitada por difusión a agregación lenta conforme aumenta el grado de recubrimiento de las partículas.

Finalmente, se procedió al cálculo del parámetro de homogeneidad $\lambda$ mediante la ecuación [13]. Los valores obtenidos están resumidos en la tabla II. Como se puede observar, el valor de $\lambda$ se mantiene prácticamente en cero para todos los recubrimientos. Únicamente para las partículas totalmente recubiertas, se detecta un ligero incremento. El parámetro $\lambda$ caracteriza el régimen de agregación dado que relaciona la velocidad de agregación entre dos partículas pequeñas con la velocidad de agregación entre partículas de mayor tamaño (ver ecuación (4)). Por lo tanto, los resultados obtenidos para $\lambda$ indican que el régimen de agregación se mantiene prácticamente inalterado aunque la velocidad del proceso se reduzca sustancialmente conforme se incrementa el grado de recubri- miento de las partículas. También se pone de manifiesto que el exponente $\alpha$ no es suficiente para caracterizar completamente un régimen de agregación y únicamente el parámetro $\lambda$ permite identificar los distintos regímenes de agregación.

\section{CONCLUSIONES}

Este trabajo presenta un estudio de la agregación de partículas coloidales cuya superficie se modificó adsorbiendo distintas cantidades de proteína. Las medidas de la velocidad de agregación demuestran que la velocidad de agregación decrece conforme aumenta el grado de recubrimiento con proteína.

Además se desarrolló un procedimiento para obtener el parámetro de homogeneidad $\lambda$ a partir de la evolución temporal de la distribución de tamaño y del tamaño medio de los agregados. Se observó que, bajo las condiciones experimentales mencionadas anteriormente, el parámetro $\lambda$ es prácticamente independiente de las propiedades superficiales de las partículas. Por lo tanto, se concluye que el régimen de agregación no se ve afectado por la presencia de la proteína en la superficie de las partículas. Únicamente para partículas totalmente recubiertas, se observa un ligero incremento en $\lambda$ que puede interpretarse como el comienzo de un cambio en el régimen de agregación.

\section{AGRADECIMIENTOS}

Este trabajo ha sido financiado por la Comisión Interministerial de Ciencia y Tecnología (CICYT, proyecto MAT97-1024). Artur Schmitt agradece a la fundación Gottlieb Daimler-und Karl Benz-Stiftung la concesión de una beca.

\section{BIBLIOGRAFÍA}

1. E. Dickinson, L. Eriksson, "Particle flocculation by adsorbing polymers", Adv. Colloid Interface Sci., 34, 1-29 (1991)

2. A. Schmitt, A. Fernández-Barbero, M. Cabrerizo-Vílchez, R. Hidalgo-Álvarez, "On the identification of bridging flocculation: An extended collision efficiency model", Prog. Colloid. Polym. Sci. 110, 105-109 (1998)

3. A. Fernández-Barbero, M. Cabrerizo-Vílchez, R. Martínez-García, R. HidalgoÁlvarez, "Effect of particle surface charge density on the colloidal aggregation mechanism", Phys Rev E, 53(5), 4981-4989 (1996)

4. M. v. Smoluchowski, "Versuch einer mathematischen Theorie der Koagulationskinetik kolloider Lösungen", Z. Phys. Chem. 92, 129-168 (1917)

5. P. G. J. Van Dongen, M. H. Ernst, “Dynamic scaling in the kinetics of clustering", J. Colloid Interface Sci. 134(1), 139-146 (1990)

6. B. J. Olivier, C. M. Sorensen, “Evolution of the cluster size distribution during slow colloidal aggregation", Phys. Rev. Lett. 54, 1396-1399 (1985)

7. A. Fernández-Barbero, A. Schmitt, M. Cabrerizo-Vílchez, R. Martínez-García, “Cluster-size distribution in colloidal aggregation monitored by single-cluster light scattering", Physica A, 230, 53-74 (1996)

8. M. L. Broide, R. J. Cohen, “Measurements of cluster-size distributions arising in salt-induced aggregation of polystyrene microspheres", J. Colloid Interface Sci. 153, 493-508 (1992)

9. A. Schmitt, A. Fernández-Barbero, M. Cabrerizo-Vílchez, R. Hidalgo-Álvarez, artículo aceptado para su publicación en un libro editado por la Editorial de la Universidad de Almería (2000)

10. A. Schmitt, A. Fernández-Barbero, M. Cabrerizo-Vílchez, R. HidalgoÁlvarez, "Experimental evidence regarding the influence of surface charge on the bridging flocculation mechanism", Prog. Colloid. Polym. Sci. 104, 144-147 (1997)

11. H. Holthoff, A. Schmitt, A. Fernández-Barbero, M. Borkovec, M. A Cabrerizo-Vílchez, P. Schurtenberger, R. Hidalgo-Álvarez, "Measurements of absolute coagulation rate constants for colloidal particles: comparison of single and multiparticle light scattering techniques", J. Colloid Interface Sci. 192, 463 (1997)

12. H. Sonntag y K. Strenge, "Coagulation Kinetics and Structure Formation", Plenum Press, New York, 1987. 